\title{
OPEN
}

\section{Author Correction: Exploring the Sequence-based Prediction of Folding Initiation Sites in Proteins}

\author{
Daniele Raimondii, ${ }^{1,3,4}$, Gabriele Orlando ${ }^{1,2,3,4}$, Rita Pancsa ${ }^{5}$, Taushif Khan ${ }^{1,3,4}$ \& \\ Wim F. Vranken $\mathbb{D}^{1,3,4}$
}

Correction to: Scientific Reports https://doi.org/10.1038/s41598-017-08366-3, published online 18 August 2017

This Article contains errors in the Methods section under subheading 'Code availability'.

"The EFoldMine code is available from https://www.dropbox.com/s/eslk4bkpflsgiia/code.zip (note: this is a temporary link, will be replaced when accepted), for academic use only as a stand-alone package with the following dependencies: Python2.7 including numpy $(>=1.6 .1)$, ScipPy $(>=0.9)$ and the scikit-learn package (http:// scikit-learn.org/stable/install.html)."

should read:

"The EFoldMine code is available from https://figshare.com/articles/EFoldMine_code/5649373, for academic use only as a stand-alone package with the following dependencies: Python2.7 including numpy $(>=1.6 .1)$, ScipPy $(>=0.9)$ and the scikit-learn package (http://scikit-learn.org/stable/install.html)."

\begin{abstract}
(c) (i) Open Access This article is licensed under a Creative Commons Attribution 4.0 International (c) License, which permits use, sharing, adaptation, distribution and reproduction in any medium or format, as long as you give appropriate credit to the original author(s) and the source, provide a link to the Creative Commons license, and indicate if changes were made. The images or other third party material in this article are included in the article's Creative Commons license, unless indicated otherwise in a credit line to the material. If material is not included in the article's Creative Commons license and your intended use is not permitted by statutory regulation or exceeds the permitted use, you will need to obtain permission directly from the copyright holder. To view a copy of this license, visit http://creativecommons.org/licenses/by/4.0/.
\end{abstract}

(C) The Author(s) 2019

\footnotetext{
${ }^{1}$ Interuniversity Institute of Bioinformatics in Brussels, ULB/VUB, Triomflaan, BC building, 6th floor, CP 263, 1050, Brussels, Belgium. ${ }^{2}$ Machine Learning Group, Université Libre de Bruxelles, Boulevard du Triomphe, CP 212, 1050, Brussels, Belgium. ${ }^{3}$ Centre for Structural Biology, VIB, Pleinlaan 2, 1050, Brussels, Belgium. ${ }^{4}$ Structural Biology Brussels, Vrije Universiteit Brussel, Pleinlaan 2, 1050, Brussels, Belgium. ${ }^{5}$ MRC Laboratory of Molecular Biology, Francis Crick Avenue, Cambridge Biomedical Campus, Cambridge, CB2 0OH, United Kingdom. Daniele Raimondi and Gabriele Orlando contributed equally. Correspondence and requests for materials should be addressed to W.F.V. (email: wvranken@vub.ac.be)
} 